\title{
The effects of prefrontal lesions on working memory performance and theory
}

\author{
CLAYTON E. CURTIS \\ New York University, New York, New York \\ and \\ MARK D'ESPOSITO \\ University of California, Berkeley, California
}

\begin{abstract}
The effects of experimental lesions of the monkey prefrontal cortex have played a predominant role in current conceptualizations of the functional organization of the lateral prefrontal cortex, especially with regard to working memory. The loss or sparing of certain performance abilities has been shown to be attributable to differences in the specific requirements of behavioral testing (e.g., spatial vs. nonspatial memoranda) along with differences in the specific locations of applied ablations (e.g., dorsal vs. ventral prefrontal cortex). Such findings, which have accumulated now for over a century, have led to widespread acceptance that the dorsolateral and ventrolateral aspects of the prefrontal cortex may perform different, specialized roles in higher order cognition. Nonetheless, it remains unclear and controversial how the lateral prefrontal cortex is functionally organized. Two main views propose different types of functional specialization of the dorsal and ventral prefrontal cortex. The first contends that the lateral prefrontal cortex is segregated according to the processing of spatial and nonspatial domains of information. The second contends that domain specialization is not the key to the organization of the prefrontal cortex, but that instead, the dorsal and ventral prefrontal cortices perform qualitatively different operations. This report critically reviews all relevant monkey lesion studies that have served as the foundation for current theories regarding the functional organization of the prefrontal cortex. Our goals are to evaluate how well the existing lesion data support each theory and to enumerate caveats that must be considered when interpreting the relevant literature.
\end{abstract}

The effects of experimental lesions of the monkey prefrontal cortex (PFC) have played a predominant role in current conceptualizations of prefrontal function, and most notably of working memory. Working memory allows organisms to use information that is not currently present in the environment but is vital to adaptive behavior. Current conceptualizations include both a maintenance component, akin to short-term memory, and an "executive" component that includes various control processes that in some way transform maintained representations into a more usable form. The loss or preservation of certain working memory abilities has been shown to be attributable to differences in the specific requirements of behavioral testing (e.g., spatial vs. nonspatial, delay vs. no delay) along with differences in the specific locations of applied ablations (e.g., dorsal vs. ventral PFC). Such findings, which have accumulated now for over a century, have led to widespread acceptance that the dorsolateral (BA 46, 9/46, and 9) and ventrolateral (BA 45

This work was supported by grants from the National Institutes of Health and the James S. McDonnell Foundation to C.E.C. Special thanks to Gregory M. Weiner for illustrating the monkeys in Figure 2. Correspondence concerning this article should be addressed to C. E. Curtis, Department of Psychology, Center for Neural Science, New York University, 6 Washington Place, Room 859, New York, NY 10003 (email: clayton.curtis@nyu.edu). and 47/12) aspects of the PFC (see Figure 1) may perform different, specialized roles in higher order cognition. ${ }^{1}$ Nonetheless, it remains unclear and controversial how the lateral PFC is functionally organized.

Two main views propose different types of functional specialization for the dorsal and ventral PFC. The first, the material-specific model, contends that the lateral PFC is segregated according to the processing of spatial and nonspatial domains of information (Levy \& GoldmanRakic, 2000). The dorsolateral PFC is engaged in "online" maintenance of spatial memoranda, while the ventrolateral PFC supports nonspatial (e.g., face, objects) memoranda. In essence, the segregation of the dorsal "where" and ventral "what" visual pathways in posterior extrastriate, parietal, and temporal cortices are proposed to be conserved in the lateral PFC. This appealing hypothesis is founded on the relatively greater proportion of projections from the dorsal stream to the dorsolateral PFC and, similarly, the greater proportion of projections from the ventral stream to the ventrolateral PFC (see Figure 1). The second, process-specific model contends that domain specialization is not the key to the organization of the PFC. This model does not deny that material or domain specificity exists in the PFC; it simply attempts to describe another functional topography. To this end, the dorsal and ventral prefrontal cortices are proposed to per- 


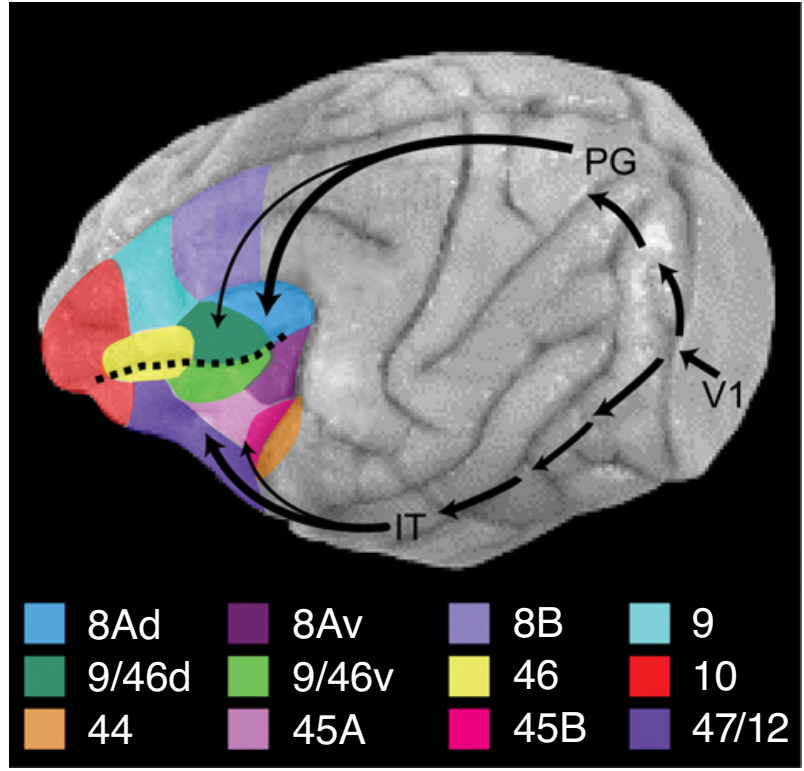

Figure 1. Subdivisions of the macaque prefrontal cortex as defined by Petrides and Pandya (1994) on the basis of connectivity and cytoarchitecture. The mid-dorsolateral PFC is composed of areas 46 and 9/46d, and the mid-ventrolateral $P F C$ of areas 47/12, $45 \mathrm{~A}$, and 9/46v. Note that many of the lesion studies reviewed here used Walker's earlier cytoarchetectonic map (Walker, 1940), in which Petrides and Pandya's (1994) subdivisions 46, 9/46d, and 9/46v are combined into a simpler area 46. Also depicted are the dorsal and ventral streams that respectively process spatial and nonspatial visual information (Ungerleider \& Mishkin, 1982). The dorsal stream has relatively greater dorsolateral PFC projections, and the ventral stream has relatively greater ventrolateral PFC projections (Petrides \& Pandya, 2002; Ungerleider Courtney, \& Haxby, 1998). Projections from the posterior parietal cortex (PG) primarily target $8 \mathrm{~A}$, and to a lesser degree $9 / 46 \mathrm{~d}$, as depicted by the thickness of the line. Projections from the inferior temporal cortex (IT) primarily target $47 / 12$, and to a lesser extent 45. The dashed line represents the principal sulcus, which delineates the dorsal and ventral aspects of the prefrontal cortex's lateral convexity.

form qualitatively different operations (Petrides, 2000b). The process-specific model proposes a hierarchy in which the ventrolateral PFC supports processes such as the active encoding and retrieval of information, and the middorsolateral PFC supports higher order "executive" control functions like the monitoring and manipulation of stored information. This report critically reviews the monkey lesion studies that have provided the foundation for current theories regarding the functional organization of the PFC.

\section{History}

Hitzig (1874) and Ferrier (1886) described over 100 years ago the effects of experimental ablations of the
PFC. Early naturalistic observations made by these experimental psychologists led them to conclude that the frontal cortex is particularly concerned with intellectual and attentional rather than sensory and motor functions. Later, more extensive comparative studies and structured behavioral methods were used to investigate the effects of frontal lesions. The Italian physiologist Bianchi (1922) concluded that removal of the frontal lobes destroyed the ability of the animal to synthesize various incoming percepts and integrate these with outgoing motor commands. In his view, the frontal cortex was the top of the sensory and motor hierarchies and was the final stage of integration. The American psychologist Franz (1907), unlike his predecessors, used structured and objective behavioral testing methods (e.g., Thorndike boxes and basic visual discrimination problems) to study the effects of frontal lobe removal. Unfortunately, though, the behavioral tests he chose proved inadequate for capturing the types of mental processes that are impaired after frontal lesions. Up to this point, Hitzig, Ferrier, Bianchi, and Franz had produced intriguing but still somewhat impressionistic work that failed to establish what role the PFC might play in high-level cognition. What was clearly lacking from their studies was a means for reliably and flexibly assessing the behavioral effects of prefrontal lesions; the pioneering researchers had noted these effects but were as yet unable to capture them. In the hands of Jacobsen (1936), Hunter's (1913) delayed-response task (Figure 2) proved to be a selective index of primate prefrontal deficit. Jacobsen's highly influential monograph detailed the delayed-response and visual discrimination capabilities of monkeys that had been given prefrontal, premotor, or temporal lobe lesions. He demonstrated that only prefrontal monkeys showed a selective and delaydependent deficit on delayed-response tasks. Delayedresponse tasks depend upon the animal's ability to maintain an internal representation over the course of a delay. At the time of choice, this internal representation must guide behavior, because no features present in the environment signal the correct response. With these ideas in mind, Jacobsen concluded that the deficit caused by prefrontal cortical damage was mnemonic in nature; specifically, the monkey's ability to use "immediate memory" to guide behavior was impaired by the prefrontal lesion.

\section{The Effects of Prefrontal Lesions on the Performance of Delayed-Response Tasks}

Following Jacobsen's (1936) pivotal demonstration of impaired delayed-response task performance after prefrontal ablations, several decades of investigations have attempted to identify exactly which area(s) of the PFC are necessary for delayed-response performance (i.e., localization) and which mental process or processes necessary for delay-response performance are impaired by damage to the PFC (i.e., functional specification). 

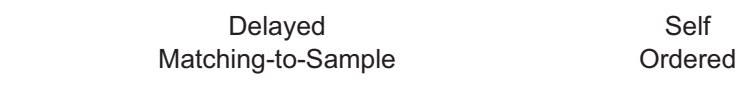

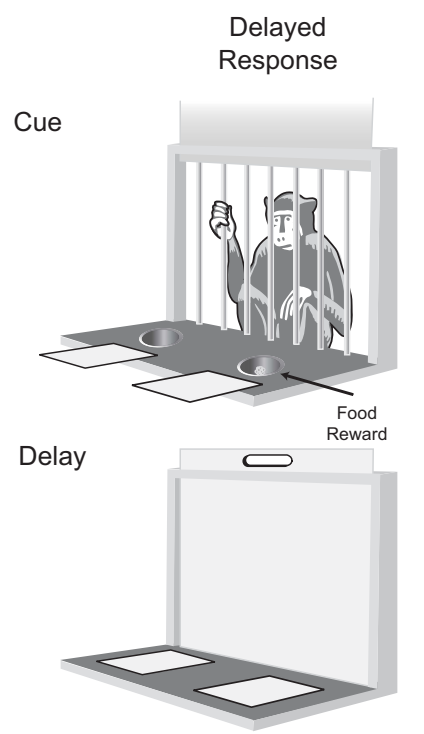

Cue

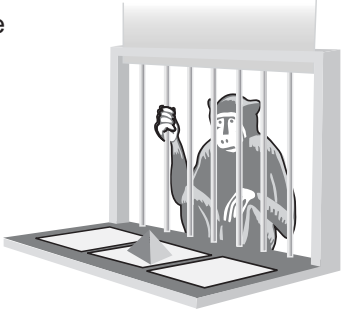

Delay
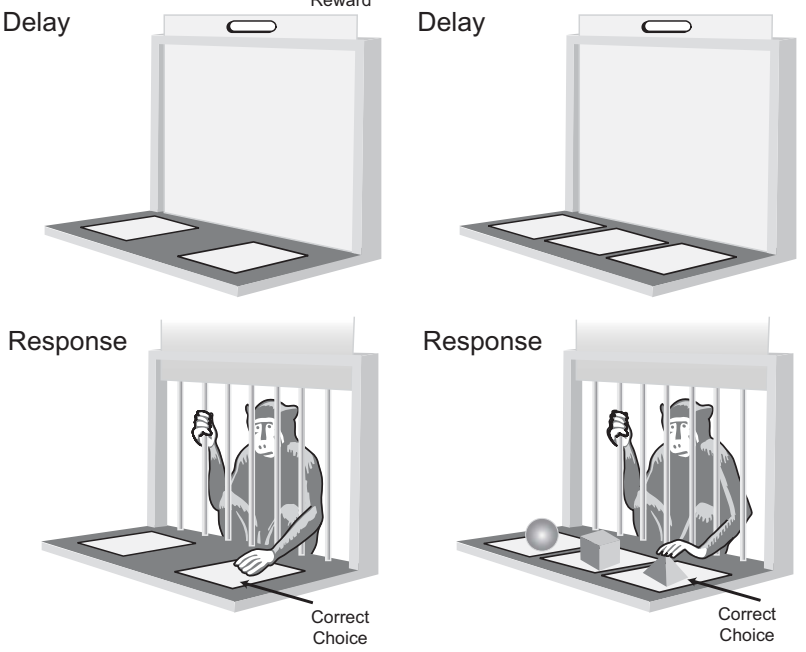

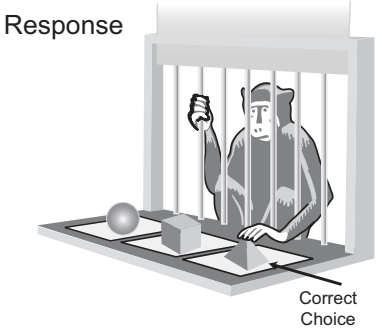

Cue

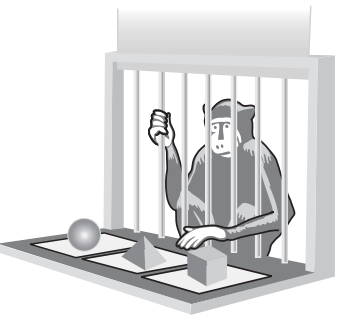

Delay
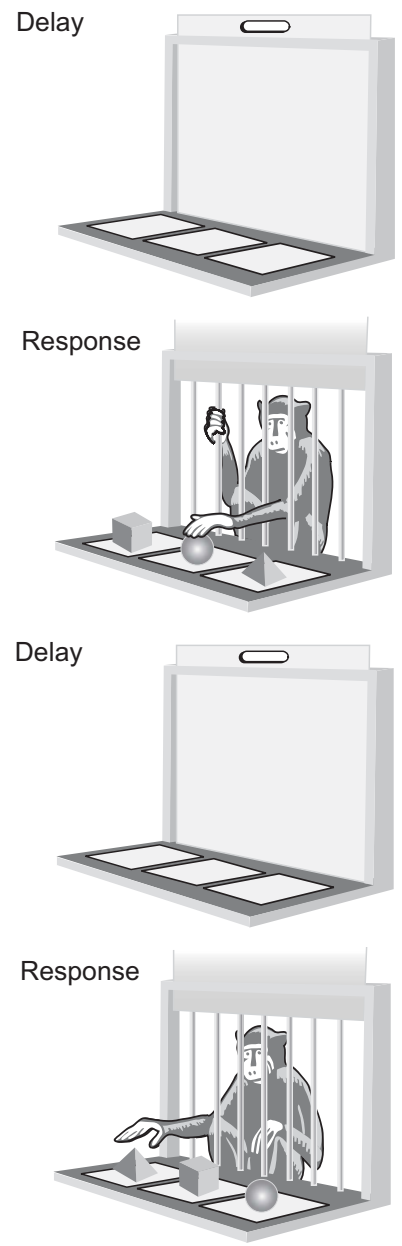

Figure 2. Schematic depictions of working memory tasks used to test the effects of prefrontal lesions. The spatial delayed-response task (left) is a test of spatial working memory and requires the monkey to remember the baited location of a food well over a delay. On a variant of this task (not shown) called delayed spatial alternation, the monkey selects the location not chosen on the immediate past trial. The delayed matching-to-sample task (middle) is a nonspatial working memory task and requires the monkey to remember an object over a delay. After the delay, only the well beneath the sample object is baited. A common variant (not shown) is the delayed nonmatching-to-sample task, in which the monkey remembers the sample object, but after the delay must select an object that does not match the sample object's form. Another nonspatial working memory variant, called delayed object alternation (not shown), is similar to spatial alternation but requires the monkey to alternatively select the object not selected on the past trial. On a self-ordered task (right), a set of objects is shown, and on each trial the monkey must select one object until they have all been selected.

\section{Localization I: The Importance of the Principal Sulcus}

Earlier studies illustrating that an intact PFC was necessary for delayed-response task performance typically made large lesions of the entire lateral prefrontal cortex, often including the medial and orbital surfaces as well. As first demonstrated by Blum (1952), an equally disabling deficit on delayed-response tasks can be produced by more focal lesions of just the "midlateral" portion of the PFC. In fact, small circumscribed lesions of just the principal sulcus (i.e., areas 46 and 9/46d) can produce deficits as great as larger total prefrontal lesions (Butters \& Pandya, 1969; Butters, Pandya, Stein, \& Rosen, 1972; Goldman \& Rosvold, 1970; Goldman, Rosvold, Vest, \& Galkin, 1971; Gross \& Weiskrantz, 1962; Mishkin, 1957). Lesions of the periacuate (i.e., areas $8 \mathrm{Ad}$ and $8 \mathrm{Av}$ ), superior frontal (i.e., areas $8 \mathrm{~B}$ and 9), orbital (i.e., areas 11 and 12), premotor (i.e., areas 6,8, and 44), inferior temporal, and parietal cortex do not typically cause significant or long-lasting impaired delayed-response performance (Butters \& Pandya, 1969; Goldman \& Rosvold, 1970; Goldman et al., 1971; Jacobsen, 1936; Rosenkilde, 
Rosvold, \& Mishkin, 1981). Importantly, lesions of the inferior convexity or ventrolateral PFC (i.e., areas $47 / 12$, $45 \mathrm{~A}$, and 45B), which are discussed below, do not produce substantial or long-lasting deficits in delayedresponse performance (Passingham, 1975). Thus, by the early 1970 s, a fair amount of evidence from lesion analyses implicated the principal sulcus as the most necessary structure required for normal performance of delayedresponse and delayed-alternation tasks (see Figure 2). The nature of the deficit caused by lesions of the principal sulcus, however, was far from being understood.

\section{Functional Specification}

Simultaneous with probes into which part of the PFC supported delayed-response task performance, investigators began asking exactly what cognitive processes were being measured. Were the deficits domain-specific (e.g., spatial vs. nonspatial memoranda) or modalityspecific (e.g., visual vs. auditory cues)? Delayed-response tasks are complex, and failure can result from a multitude of impaired processes. Were the deficits most consistent with impaired stimulus discrimination? Did they seem attributable to alterations in motor control? Or, as Jacobsen (1936) speculated, were they attributable to impairments in the ability to maintain an internal representation over a period of time? To seek answers, variations on the classic delayed-response task were conceived.

The importance of space and time. In the classical versions of the delayed-response and delayed-alternation problems, an animal must use spatial factors to solve them correctly. For the delayed-response task, the spatial position of the baited well must be remembered over a delay. Similarly, for the delayed-alternation task, the spatial position of the well chosen on the last trial must be remembered. Besides their spatial position, the two wells are otherwise indistinguishable (e.g., same color, shape, etc.). We have already seen that prefrontal lesions impair performance on such spatial delayed-response and delayed-alternation tasks. To address whether the same pattern would emerge if the animal must rely on nonspatial information, Mishkin and Pribram conducted a series of studies (Mishkin, 1954; Mishkin \& Pribram, 1954). They trained monkeys to perform delayed-response and delayed-alternation tasks that were modified so that monkeys were required to use nonspatial information to perform correctly. Remarkably, the monkeys with prefrontal lesions were able to perform these versions normally but still performed poorly on tasks for which spatial information was necessary. Therefore, the deficit following prefrontal damage appeared limited to the spatial domain. However, Mishkin and Pribram then discovered that monkeys with prefrontal lesions were just as impaired on a delayed object alternation task as on the spatial version (Mishkin \& Pribram, 1955, 1956; Pribram \& Mishkin, 1956), calling into question the domain specificity of the lesion. For the object alternation task, animals alternately chose between two easily distinguish- able objects on each trial; the positions of the objects were random and did not guide correct performance. In a followup study with prefrontal lesions limited to the dorsal and sparing the ventral lateral prefrontal surface, there was a marked deficit on spatial but not object delayedalternation tasks (Mishkin, Vest, Waxler, \& Rosvold, 1969); this effect was later replicated by Petrides (1995). Thus, damage to the dorsolateral PFC caused alternation deficits when spatial but not object information must be used to guide behavior.

In a pair of influential studies, Goldman-Rakic and colleagues (Goldman \& Rosvold, 1970; Goldman et al., 1971) presented evidence that spatial factors are necessary but not sufficient to cause deficits in monkeys with principal sulcus lesions; the imposition of a delay between cue and response is also necessary. Monkeys with lesions of the principal sulcus could not perform spatial delayed-response (Goldman et al., 1971) or spatial delayed-alternation tasks (Goldman \& Rosvold, 1970). However, these monkeys performed normally on a conditional position response task that did not impose an intratrial delay: The monkeys were rewarded for choosing a left or right box depending on a sound that originated from above or below the chamber (Goldman \& Rosvold, 1970). This task contained a spatial component but no delay. Additionally, in a study that replicated the findings of Mishkin and Pribram (1954), lesions of the principal sulcus did not cause impairments in a delayed go/no-go alternation task, which contained a delay but not a spatial component (Goldman et al., 1971). The researchers argued that in order for a behavioral task to be sensitive to lesions of the principal sulcus, it must necessarily contain both a spatial and a delay factor rather than either of these factors alone.

Other compelling ways have also been found to demonstrate that the presence of a delay is crucial. For instance, instead of comparing performance on different tasks that have or do not have delays, the same task can be used and performance can be assessed for varying delays. M. H. Miller and Orbach (1972) demonstrated that monkeys with dorsolateral PFC lesions show a delaydependent spatial alternation deficit: The monkeys can perform spatial alternation tasks when there is no delay, show mild deficits at a minimal 1-sec delay, and show pronounced deficits when the delay is lengthened to 5-sec. Similar delay-dependent effects on delayed-response tasks have been observed after cooling of the PFC (Bauer \& Fuster, 1976; Fuster \& Alexander, 1970).

Most notably, using an eight-position oculomotor delayed-response task with restrained monkeys, Funahashi, Bruce, and Goldman-Rakic (1993) were able to demonstrate convincingly that lesions of the principal sulcus interfere, in a delay-dependent manner, with the accuracy of saccades made to remembered locations of briefly presented cues in the contralesional hemifield. The deficits did not appear to be strictly sensory or motor in nature, since the monkeys had no difficulties detecting visual cues and making accurate saccades to them, as 
long as they were still visible. In fact, significant deficits were rarely noted at a brief delay of $1.5 \mathrm{sec}$ but became progressively worse as the delay was lengthened, showing a delay dependency. Funahashi and colleagues had previously shown that directionally selective delay-period neuronal activity, as measured by unit recordings of cellular activity in the principal sulcus in awake-behaving monkeys performing oculomotor delayed-response tasks, may represent maintenance of the spatial cue over the delay (Funahashi, Bruce, \& Goldman-Rakic, 1989, 1990, 1991). Also notably, Funahashi et al. (1993) reported for the first time impairments when only one PFC hemisphere was lesioned. Past studies done in the Wisconsin General Testing Apparatus, a testing chamber in which the monkey was free to move its head during the testing, had not found significant impairments unless both PFC hemispheres were damaged. However, with the monkey's head restrained and eye position controlled, Funahashi et al. (1993) were able to demonstrate that unilateral lesions were sufficient to cause contralesional impaired spatial working memory performance (see Figure 3 ). Together, these data strongly link the principal sulcus to the maintenance of contralateral spatial information.

Non-spatial working memory performance after dorsolateral prefrontal lesions. In the 1970s, Fuster and his colleagues began experimenting with localized cryogenic depressing or "cooling" of cortical tissue as a means of inducing a reversible lesion. These investigators demonstrated that cooling of the principal sulcus impaired performance of not only spatial delayedresponse tasks (Bauer \& Fuster, 1976; Fuster \& Alexander, 1970; Fuster \& Bauer, 1974) but also nonspatial delayed matching-to-sample tasks (Bauer \& Fuster, 1976;
Quintana \& Fuster, 1993). These data argue against the hypothesis that the principal sulcus supports short-term memory for spatial information only and suggests that it has a more general role in working memory. To bolster this claim, Fuster and others have cited unit recording studies in which delay period activity of neurons in the principal sulcus is just as great during spatial as during nonspatial working memory tasks (Fuster, Bauer, \& Jervey, 1982; Quintana \& Fuster, 1992; Quintana, Yajeya, \& Fuster, 1988; Rao, Rainer, \& E. K. Miller, 1997).

Other investigations around the same time showed that lesions limited to the principal sulcus do not cause nonspatial delayed alternation or delayed matching-to-sample deficits (Mishkin \& Manning, 1978; Mishkin et al., 1969; Passingham, 1975; Stamm, 1973; Stamm \& WeberLevine, 1971), but lesions ventral to the principal sulcus do cause such impairments severely (Mishkin \& Manning, 1978; Passingham, 1975). Moreover, further studies have confirmed that lesions of the dorsolateral PFC do not cause impaired nonspatial working memory (Bachevalier \& Mishkin, 1986; Levy \& Goldman-Rakic, 1999; Petrides, 1991, 1995, 2000a). How do we reconcile these discrepancies? Some investigators attributed the nonspatial working memory deficits noted after cooling of the principal sulcus to a spreading of the cooling to adjacent prefrontal cortical tissue. Although cryogenic depression does result in the dampening of synaptic activity, the effects are not very localizable, and there is even a halo zone around the cooled center that can exhibit hyperactive synaptic activity (Volgushev, Vidyasagar, Chistiakova, \& Eysel, 2000). This spatial uncertainty, combined with recent studies that show that dorsolateral PFC lesions do not cause impaired maintenance of object stimuli (Levy \&

\section{A. Oculomotor Delayed-Response Task}

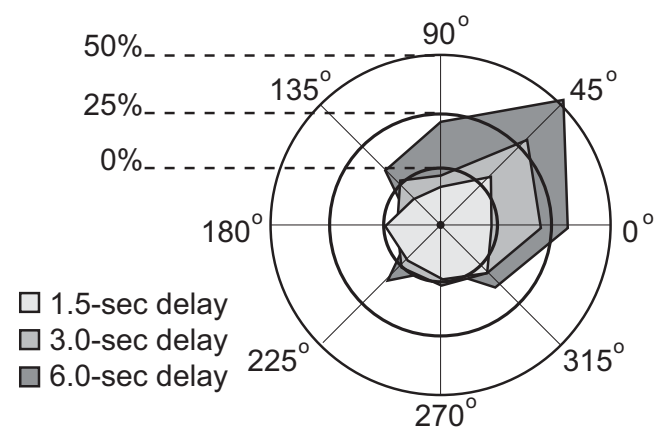

B. Oculomotor Control Task

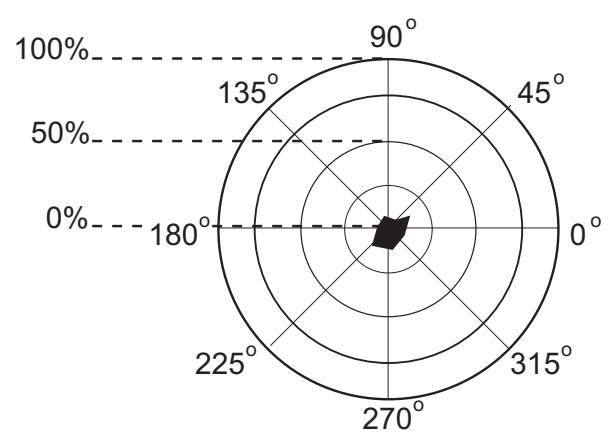

Figure 3. Unilateral lesion to the principal sulcus causes impaired memory for spatial locations in the contralesional hemifield. Example data are shown from an oculomotor version of a spatial delayedresponse task, in which monkeys made saccades to the remembered location of cues. (A) Accuracy of memory-guided saccade is impaired for locations in the upper right quadrant after left principal sulcus lesion. Note that the lengthening of the delay exacerbates the deficit. Accuracy is depicted as a percentage of decline in accuracy after the lesion. (B) Accuracy of visually guided saccades is not impaired, suggesting intact visual and motor function. From "Dorsolateral prefrontal lesions and oculomotor delayedresponse performance: Evidence for mnemonic 'scotomas,"' by S. Funahashi, C. J. Bruce, and P. S. Goldman-Rakic, 1993, Journal of Neuroscience, 13, p. 1482. Copyright 1993 by the Society for Neuroscience. Adapted with permission. 
Goldman-Rakic, 1999; Petrides, 2000a), cause us to conclude that dorsolateral PFC lesions cause specific impairments in the maintenance of spatial memoranda.

Sensory modality of memoranda is not limited to vision. Almost all of the studies we have discussed up to this point have used visually presented stimuli, which leaves open the possibility that the deficits they have noted following prefrontal damage are modality specific. However, this does not appear to be the case. Prefrontal lesions also cause delayed-response impairments when spatial memoranda cues are auditory (Blum, 1952; Oscar-Berman, 1975). Cooling of the PFC also causes impairments when memoranda are perceived haptically (Shindy, Posley, \& Fuster, 1994). Therefore, it appears that lesions to the PFC cause impaired delayed-response performance regardless of the input sensory modality.

Monitoring and self-ordered task performance after dorsolateral prefrontal lesions. Michael Petrides, the major proponent of the process-specific theory of midlateral PFC organization, developed a variation on the delayed-response task called the self-ordered task (see Figure 2; Petrides \& Milner, 1982). Self-ordered tasks not only require that the animal remember some stimulus feature over a delay but also critically tax the animal's ability to monitor the contents of working memory. Monitoring, according to Petrides (Petrides, 2000b), is an executive control process that is used to track, update, and order the number of items stored in working memory. According to the process-specific model, the middorsolateral PFC (i.e., area 9/46d and 46) supports this executive process irrespective of the nature of the material (i.e., spatial vs. nonspatial). Recall that monkeys with dorsolateral PFC lesions are not impaired on tests of nonspatial working memory that rely on recognition memory (e.g., delayed matching or nonmatching-tosample or object alternation tasks). However, they do fail on nonspatial self-ordered tasks that in addition to maintenance, require the executive process of monitoring (Petrides, 1991, 1995, 2000a). These results are the cornerstone of the process-specific model's position that the mid-dorsolateral PFC supports working memory processes, both spatial and nonspatial, by tracking or monitoring this information.

Levy and Goldman-Rakic (1999) directly challenged these conclusions with data from monkeys with dorsolateral PFC lesions that performed spatial and nonspatial versions of self-ordered and delayed-response or matching tasks. Lesions to the dorsolateral PFC caused impairments on spatial delayed-response tasks but not on object delayed nonmatching-to-sample tasks, replicating the effects that had been shown many times before (see above). Lesions to the dorsolateral PFC caused lasting impairments on the spatial but not the object selfordered task. Animals had not been tested on a spatial version of the self-ordered task previous to this study, but impairments on such a task are consistent with both the material- and process-specific models. However, the intact performance of monkeys with lesions to the dorsolateral PFC on the object version of the self-ordered task, a version very similar to the one that yielded deficits in Petrides's studies, is particularly problematic (Levy \& Goldman-Rakic, 2000).

These data are strong evidence in favor of the materialspecific model, at least with regard to the specific role of the dorsolateral PFC in spatial working memory. The comparability of the exact location of the dorsolateral PFC lesions in Petrides (2000b) and Levy and GoldmanRakic (1999) must be carefully considered, however. Both sets of investigators acknowledged that the lesioned loci were not the same and that this fact could be related to the discrepancy in their results (see Figure 4). Levy and Goldman-Rakic (1999) lesioned the tissue in the depths of the sulcus in areas $46,9 / 46 \mathrm{~d}$, and the anterior portion of sulcal 8Ad. The Petrides (2000b) lesions were mostly restricted to the dorsal lip of the principal sulcus extending up around the midlateral gyrus (areas 46, 9/46d, and inferior-lateral portions of area 9), but importantly spared most of the tissue in the depths of the sulcus itself. Therefore, it may be that intact performance on the object self-ordered task can be seen after damage to the mid-dorsolateral PFC, as long as the lesion spares the gyral convexity.

Dorsolateral prefrontal cortex lesion summary. Overall, there is compelling evidence, much of it from the work of Goldman-Rakic, that damage to the dorsolateral PFC, especially in the depths of the principal sulcus in areas 46, 9/46, and 8Ad, causes profound impairments in spatial working memory. Performance is typically intact on tests of nonspatial working memory unless executive control processes are required, as has been demonstrated by the work of Petrides. Several outstanding issues have yet to be resolved, however: First, the differential contributions of the sulcal and gyral portions of the dorsolateral PFC to working memory performance need to be addressed. For instance, it is unknown whether or not lesions like those in Petrides (2000b), but extending to the convexity of the mid-dorsolateral PFC in areas 46 and 9/46, would result in impaired performance on a spatial delayed-response task, which does not depend critically on monitoring. Second, how much does impaired spatial working memory depend on damage to area 8Ad, which is known to contain the frontal eye fields and was included in the lesions in Levy and Goldman-Rakic (1999)? The second question has been partially addressed by Funahashi et al. (1993), in which two of the monkeys' lesions did not extend into caudal 8Ad; these monkeys did indeed show impaired oculomotor delayed-response task performance.

\section{Localization II: Ventrolateral Prefrontal Cortex (or Inferior Convexity)}

As discussed, lesions of the principal sulcus cause impaired spatial but unimpaired nonspatial working memory deficits, a pattern of findings consistent with the 
A
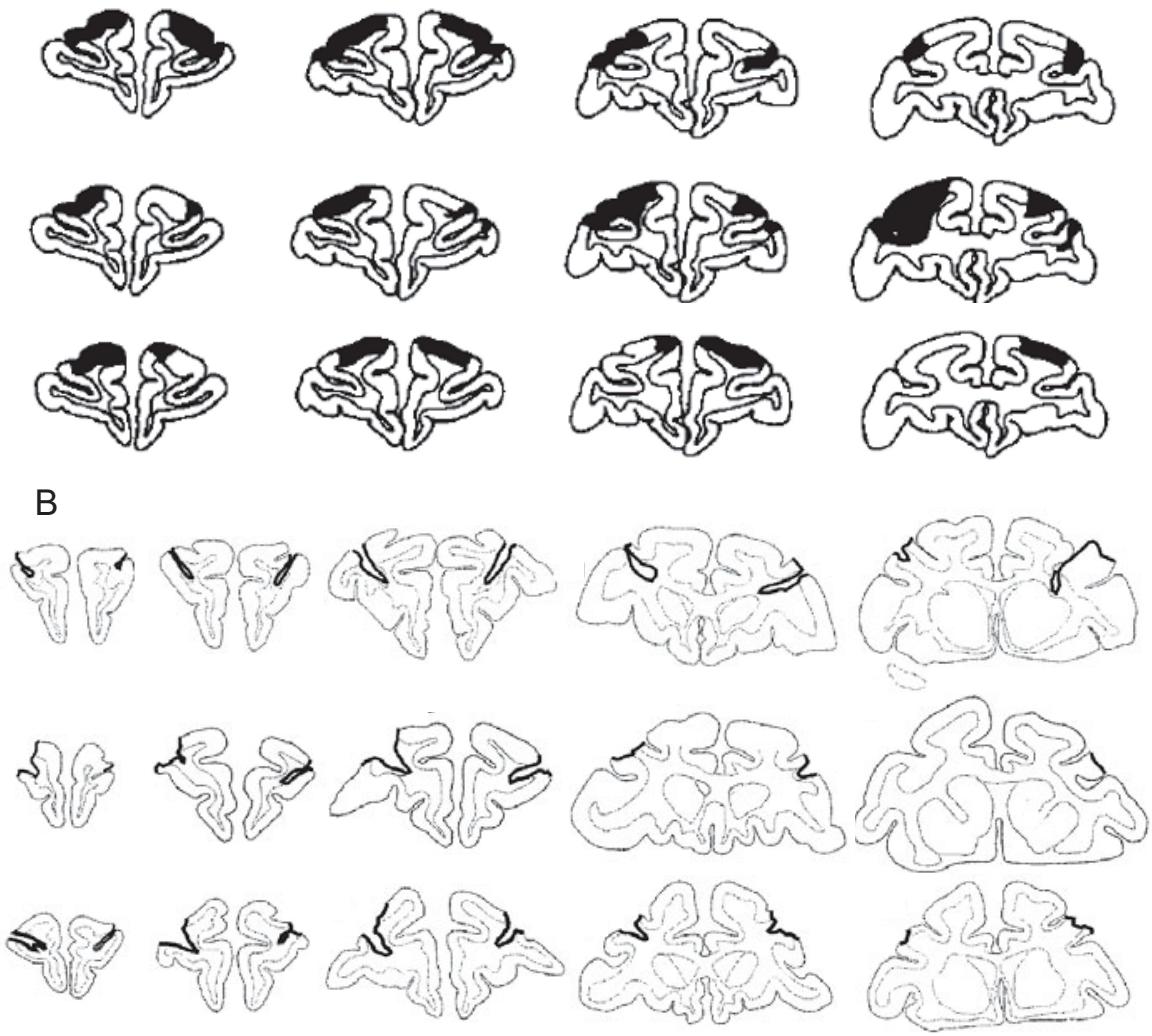

Figure 4. Lesions from (A) Petrides (2000a) and (B) Levy and Goldman-Rakic (1999). Levy and Goldman-Rakic (1999) lesioned the tissue in the depths of the sulcus in areas 46, 9/46d, portions of 9/46v, and the anterior portion of sulcal 8Ad. The Petrides (2000a) lesions were mostly restricted to the dorsal lip of the principal sulcus extending up around the midlateral gyrus (areas 46, 9/46d, and inferior-lateral portions of area 9), but spared most of the tissue in the depths of the sulcus itself. (A) From "Dissociable roles of mid-dorsolateral prefrontal and anterior inferotemporal cortex in visual working memory," by M. Petrides, 2000, Journal of Neuroscience, 20, p. 7497. Copyright 2000 by the Society for Neuroscience. Reprinted with permission. (B) From "Association of storage and processing functions in the dorsolateral prefrontal cortex of the nonhuman primate," by R. Levy and P. S. Goldman-Rakic, 1999, Journal of Neuroscience, 19, p. 5152. Copyright 1999 by the Society for Neuroscience. Reprinted with permission.

material-specific model. In addition, that model proposes that the tissue below the principal sulcus (areas $46 \mathrm{v}, 9 / 46 \mathrm{v}, 47 / 12$ ) is specialized for the maintenance of nonspatial memoranda, like objects and faces. Indeed, neurons in area 45 and part of area 12 often show selective responses to visually presented faces and objects (Ó Scalaidhe, Wilson, \& Goldman-Rakic, 1999; Wilson, Ó Scalaidhe, \& Goldman-Rakic, 1993). In contrast with the dorsolateral PFC literature, relatively little data has accumulated on the effects of lesions to the ventrolateral PFC, but nonetheless we review these findings below.

Spatial working memory performance after ventrolateral prefrontal lesions. Contrary to the common claim that lesions to the ventrolateral PFC do not normally impair spatial working memory (e.g., Levy \& Goldman-Rakic, 1999, 2000), substantial impairments on tests of spatial working memory have often been observed following such lesions (Iversen \& Mishkin, 1970; Mishkin et al., 1969; Passingham, 1975; Rosenkilde et al., 1981).

Nonspatial working memory performance after ventrolateral prefrontal lesions. Lesions to the ventrolateral PFC have been shown to produce impaired performance on tests of nonspatial working memory, like delayed object alternation tasks (Mishkin \& Manning, 1978) and tests of recognition memory for objects or colors (Iversen \& Mishkin, 1970; Mishkin \& Manning, 1978; Passingham, 1975). However, these impairments are often reported to be relatively mild and transient (Kowalska, Bachevalier, \& Mishkin, 1991; Passingham, 1975). Also, unlike the results following dorsolateral PFC lesions, where performance deficits necessarily de- 
A

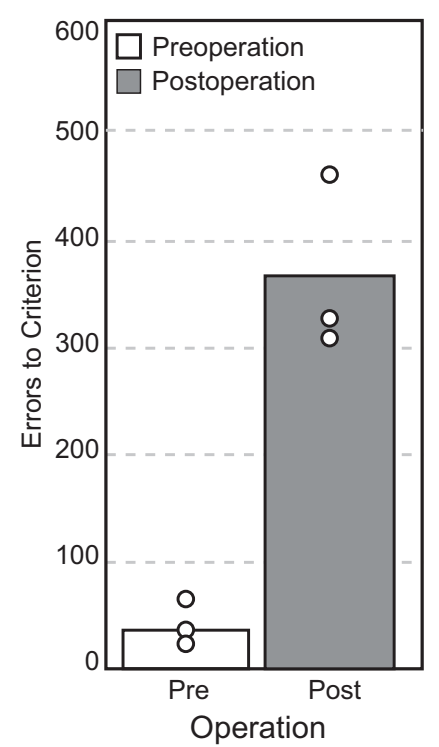

B

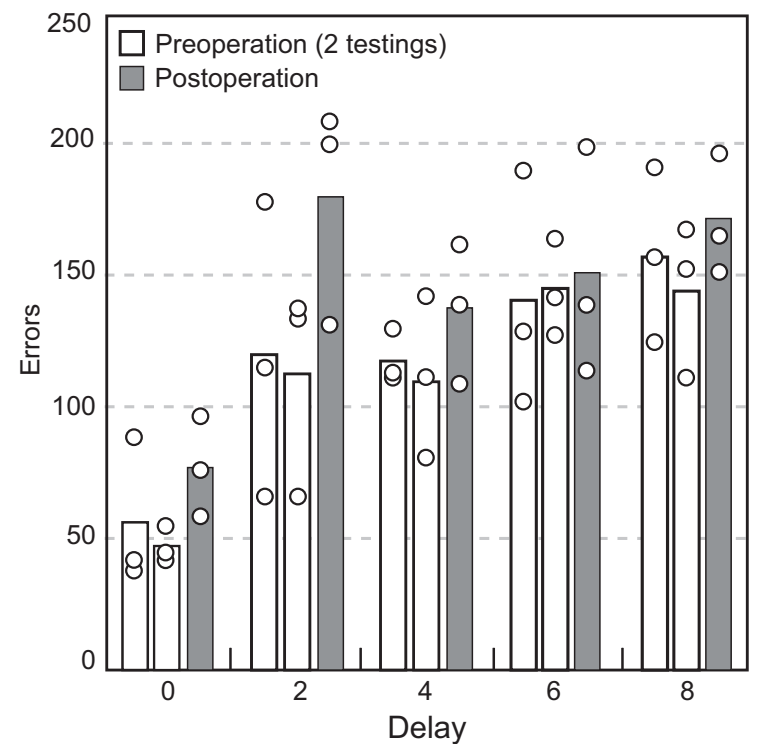

Figure 5. Results of lesions to the ventrolateral PFC (areas 47/12 and 45A) on a color matchingto-sample task. (A) No-delay matching. Number of errors made during task learning before and after lesion. The sample cue and the two color choices were presented simultaneously with no delay. (B) Delayed matching. Number of errors made at various delays before and after lesion. After relearning the task with extended training, the monkeys were retested with a delayed matching-tosample task. Note that the introduction of a delay did not interact with the lesion. From "Ventral prefrontal cortex is not essential for working memory," by M. F. Rushworth, P. D. Nixon, M. J. Eacott, and R. E. Passingham, 1997, Journal of Neuroscience, 17, pp. 4835-4836. Copyright 1997 by the Society for Neuroscience. Adapted with permission.

pend on the imposition of a delay, ventrolateral PFC lesions often cause impairments even when no delay is present (Iversen \& Mishkin, 1970; Passingham, 1975; Rushworth, Nixon, Eacott, \& Passingham, 1997). Indeed, no delay-dependent working memory impairment has been reported following ventrolateral PFC lesions.

The most compelling data that questions the role of the ventrolateral PFC in working memory generally, and not just in nonspatial working memory, is that of Rushworth et al. (1997), who reported that removal of the ventrolateral PFC (area 47/12) resulted in no effect on pattern or color working memory. Lesions were then extended to include area 45A, which resulted in impairments in a color matching-to-sample task with no delay (i.e., the sample and response choices were presented simultaneously), but the imposition of increasing delays did not exacerbate the effect of the lesion (see Figure 5). These data led the authors to suggest that the ventrolateral PFC is not essential for working memory.

Associative learning after ventrolateral prefrontal lesions. Given that the effects of lesions to the ventrolateral PFC may not be best characterized as impaired working memory, what role may this portion of the cortex have in cognition? There have been several reports of impaired performance on what are called conditional visuomotor learning or arbitrary visuomotor mapping tasks after lesions to the ventrolateral PFC, which thus deserve comment. These tasks require that the animal learn arbitrary associations between visual cues and motor responses. The word "arbitrary" is important here, because it means that the stimulus-response mapping is not spatially guided. For instance, the position of a red light at an intersection does not in itself provide any guide as to where one should drive, but it does indicate that one should stop; there is an arbitrary nonspatial mapping between a red signal light and the motor behavior associated with the light.

Using a crossed disconnection procedure, in which the ventrolateral PFC is damaged in one hemisphere and the inferior temporal (IT) cortex is damaged in the other hemisphere followed by a transection of the corpus callosum, Gaffan and colleagues have shown that interactions between the ventrolateral PFC and IT are necessary for normal learning of arbitrary visuomotor associations (Gaffan \& Harrison, 1988; Parker \& Gaffan, 1998). Transection of the major fiber bundle connecting IT and ventrolateral PFC, the uncinate fasciculus, also impairs learning of arbitrary visuomotor associations (Eacott \& Gaffan, 1992). Moreover, monkeys with bilateral ventrolateral PFC lesions have terrible difficulties learning arbitrary visuomotor mappings (Bussey, Wise, \& Murray, 2001; see Figure 6). These deficits do not appear to be caused by sensory or motor failures per se, as the monkeys can achieve substantial levels of performance 


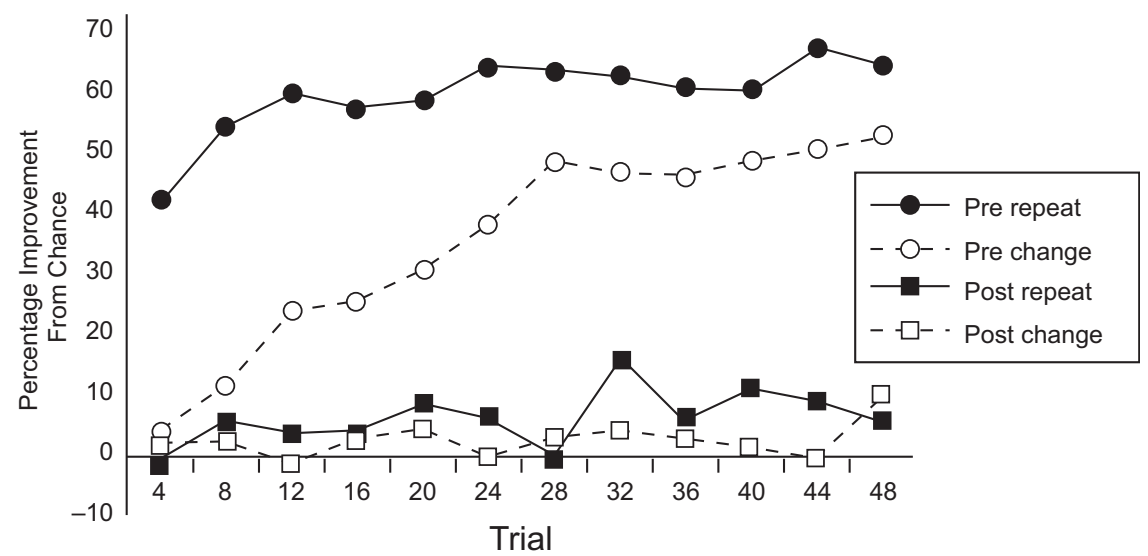

Figure 6. Effects of ventrolateral PFC lesions on conditional visuomotor learning. Monkeys attempted to learn an arbitrary joystick movement (left/right/pull) associated with three visual cues. On repeat trials the visual cue was the same as on the immediately preceding trial (filled markers), but on change trials the visual cue was different (open markers). Prior to the lesion, monkeys learned the visuomotor associations, as can be seen by the increasing slope $($ Pre $=$ preoperative $)$. Since performance is already above chance very early in the session, before the associations have been learned, the monkeys must have appreciated the strategies of repeat-stay and change-shift. After the lesion, the monkeys showed no evidence of learning the visuomotor associations; performance was at chance levels (Post $=$ postoperative). Additionally, they failed to use adaptive strategies: On repeat trials, they failed to emit the same response after correctly responding (repeat-stay), and on change trials, they failed to emit a different response after correctly responding (change-shift). From "The role of ventral and orbital prefrontal cortex in conditional visuomotor learning and strategy use in rhesus monkeys (Macaca mulatta), by T. J. Bussey, S. P. Wise, and E. A. Murray, 2001, Behavioral Neuroscience, 115, p. 975. Public domain.

on a concurrent visual discrimination task, and analysis of the errors indicates that they are not consistent with perseveration or systematic response biases (Bussey et al., 2001). Even small reversible lesions applied with the local infusion of bicuculline into a restricted area of the ventrolateral PFC cause impaired arbitrary visuomotor learning, while infusions into the dorsolateral PFC do not (Wang, Zhang, \& Li, 2000). The pattern of performance deficits following ventrolateral PFC damage may be due to a failure to form and/or use arbitrary visuomotor associations. These stimulus-response mappings are essentially rules (Murray, Bussey, \& Wise, 2000; Wise $\&$ Murray, 2000) that could be low-level (for instance, stop at a red signal light), or high-level (for instance, signal lights guide traffic flow) in nature. Alternatively, the ventrolateral PFC lesion could impair performance by impacting the animal's strategic approach to the problem: Error analyses indicate that the monkeys fail to apply win-stay, lose-shift, and change-shift strategies during the learning sessions (Bussey et al., 2001; Wang et al., 2000).

Ventrolateral prefrontal lesion summary. Some evidence suggests that lesions to the ventrolateral PFC cause nonspatial working memory impairments. However, there are several problems with this interpretation: First, no study has demonstrated a selective deficit in the spatial as compared with the nonspatial domain. When deficits were found in the spatial domain, they were noted in the nonspatial domain as well. Second, there has been no demonstration of a delay dependency. Impairments have been observed with tasks that do not require maintenance, and increasing the delay does not exacerbate the deficit. Third, damage to the ventrolateral PFC causes impairments with tasks that make no working memory demands. For instance, performance on conditional visuomotor association tasks is impaired after ventrolateral PFC damage (Bussey et al., 2001; Gaffan \& Harrison, 1988; Parker \& Gaffan, 1998; Wang et al., 2000). Therefore, impairments following ventrolateral PFC lesions may be best conceptualized as an inability to form and/or use associations between cues and motor responses or may be more generally associated with inability to use learning strategies (Murray et al., 2000).

\section{Evaluating the Theories of the Functional Segregation of the Mid-Lateral PFC}

\section{Material-Specific Model}

This model, proposed by Goldman-Rakic, predicts that lesions to the dorsolateral PFC cause specific impairments in spatial, but not in nonspatial, working memory performance. Indeed, the specificity of dorsolateral PFC lesions to spatial working memory is the strongest support for this model. Lesions to the dorsolateral PFC reli- 
ably cause significant impairments in spatial working memory performance, and the effect is exacerbated with increasing delays. The model also predicts that ventrolateral PFC lesions cause specific impairments in nonspatial working memory performance. So far, very little support exists that indicates that the ventrolateral PFC is necessary for nonspatial working memory. Clearly, the overall pattern of results does not suggest a double dissociation between dorsolateral and ventrolateral PFC lesions and spatial and nonspatial working memory.

\section{Process-specific model}

This model, proposed by Petrides, predicts that lesions to the mid-dorsolateral PFC cause an impaired ability to maintain and monitor or track multiple items in working memory. The strongest support for this prediction is the finding that when monitoring demands are increased, even when the memoranda are objects, the performance of monkeys with mid-dorsolateral PFC lesions is impaired. However, these same monkeys can still maintain objects over varying retention intervals without problem. If monitoring is indeed the critical process that depends on mid-dorsolateral PFC integrity, then it remains unclear why such lesions cause impairments on simpler tests of spatial working memory; dorsolateral PFC lesions impair performance on spatial delayed response and oculomotor delayed response tasks that do not depend on monitoring. The model also predicts that ventrolateral PFC lesions will cause impairments in active or strategic encoding and retrieval of information. However, these aspects of the model have yet to be tested, and their relation to learning arbitrary visuomotor associations has yet to be formalized.

\section{Conclusions}

The material- and process-specific theories have been critical catalysts in our attempts to understand the functional specializations of PFC subregions by motivating research and providing a heuristic framework within which hypotheses can be tested. It cannot be overstated how important the findings from monkey lesion studies have been in the development of these two theories of the functional organization of the midlateral PFC. The use of lesions to map structure-function relationships existed before the development of new imaging techniques, when the roots of the current PFC theories were also developing. Recently, the lessons from these studies have no longer been given primary importance, despite the fact that lesions can be used to test the necessity of a brain region for cognition powerfully. Theory testing is currently being done with the use of correlative techniques such as electrophysiology (Funahashi, 2001; Fuster, 2001; E. K. Miller, 2000; Wilson et al., 1993) and neuroimaging (Curtis \& D'Esposito, 2003; Curtis, Zald, Lee, \& Pardo, 2000; D'Esposito, Postle, \& Rypma, 2000; Funahashi, 2001; Fuster, 2001; E. K. Miller, 2000; Owen, 2000; Owen et al., 1998; Passingham, Toni, \& Rushworth, 2000; Ungerleider, Courtney, \& Haxby, 1998; Wilson et al., 1993). The goal of this review was to revive awareness of the important contributions that lesion studies have made. To this end, we summarized the effects of PFC lesions with special emphasis on how the findings fit with predictions made by dominant PFC theories. We conclude that the most consistent results from numerous lesions to the PFC suggest that the mid-dorsolateral PFC is necessary for some aspect of spatial working memory task performance and that the ventrolateral PFC is necessary for some aspect of learning arbitrary stimulus-response associations. These conclusions only partially overlap with the predictions from the material- and process-specific models, indicating that in their current forms, neither of these models can adequately account for the functional topography of the midlateral PFC.

\section{REFERENCES}

Bachevalier, J., \& Mishkin, M. (1986). Visual recognition impairment follows ventromedial but not dorsolateral prefrontal lesions in monkeys. Behavioral \& Brain Research, 20, 249-261.

Bauer, R. H., \& Fuster, J. M. (1976). Delayed-matching and delayedresponse deficit from cooling dorsolateral prefrontal cortex in monkeys. Journal of Comparative \& Physiological Psychology, 90, 293-302.

BIANCHI, L. (1922). The mechanism of the brain and the function of the frontal lobes. Edinburgh: E. \& S. Livingstone.

BLUM, R. A. (1952). Effects of subtotal lesions of frontal granular cortex on delay reaction in monkeys. Archives of Neurology \& Psychiatry, 67, 375-386.

Bussey, T. J., Wise, S. P., \& Murray, E. A. (2001). The role of ventral and orbital prefrontal cortex in conditional visuomotor learning and strategy use in rhesus monkeys (Macaca mulatta). Behavioral Neuroscience, 115, 971-982.

ButTERS, N., \& PANDYA, D. (1969, September 19). Retention of delayedalternation: Effect of selective lesions of sulcus principalis. Science, 165, 1271-1273.

Butters, N., PANDYA, D., Stein, D., \& Rosen, J. (1972). A search for the spatial engram within the frontal lobes of monkeys. Acta Neurobiologiae Experimentalis, 32, 305-329.

CURTIS, C. E., \& D'Esposito, M. (2003). Persistent activity in the prefrontal cortex during working memory. Trends in Cognitive Sciences, 7, 415-423.

CURTIS, C. E., Zald, D. H., Lee, J. T., \& Pardo, J. V. (2000). Object and spatial alternation tasks with minimal delays activate the right anterior hippocampus proper in humans. NeuroReport, 11, 2203-2207.

D'Esposito, M., Postle, B. R., \& Rypma, B. (2000). Prefrontal cortical contributions to working memory: Evidence from event-related fMRI studies. Experimental Brain Research, 133, 3-11.

EACотт, M. J., \& GAFFAN, D. (1992). Inferotemporal-frontal disconnection: The uncinate fascicle and visual associative learning in monkeys. European Journal of Neuroscience, 4, 1320-1332.

Ferrier, D. (1886). The functions of the brain (2nd. ed.). London: Smith, Elder, \& Co.

FRANZ, S. I. (1907). On the functions of the cerebrum: The frontal lobes. New York: Science Press.

FUNAHASHI, S. (2001). Neuronal mechanisms of executive control by the prefrontal cortex. Neuroscience Research, 39, 147-165.

Funahashi, S., Bruce, C. J., \& Goldman-Rakic, P. S. (1989). Mnemonic coding of visual space in the monkey's dorsolateral prefrontal cortex. Journal of Neurophysiology, 61, 331-349.

Funahashi, S., Bruce, C. J., \& GoldmaN-RaKic, P. S. (1990). Visuospatial coding in primate prefrontal neurons revealed by oculomotor paradigms. Journal of Neurophysiology, 63, 814-831.

Funahashi, S., BRuce, C. J., \& Goldman-R AKIC, P. S. (1991). Neu- 
ronal activity related to saccadic eye movements in the monkey's dorsolateral prefrontal cortex. Journal of Neurophysiology, 65, 14641483.

Funahashi, S., Bruce, C. J., \& Goldman-Rakic, P. S. (1993). Dorsolateral prefrontal lesions and oculomotor delayed-response performance: Evidence for mnemonic "scotomas." Journal of Neuroscience, 13, 1479-1497.

FUSTER, J. M. (2001). The prefrontal cortex - an update: Time is of the essence. Neuron, 30, 319-333.

Fuster, J. M., \& ALEXANDER, G. E. (1970). Delayed response deficit by cryogenic depression of frontal cortex. Brain Research, 20, 85-90.

FUSTER, J. M., \& BAUER, R. H. (1974). Visual short-term memory deficit from hypothermia of frontal cortex. Brain Research, 81, 393-400.

Fuster, J. M., Bauer, R. H., \& Jervey, J. P. (1982). Cellular discharge in the dorsolateral prefrontal cortex of the monkey in cognitive tasks. Experimental Neurology, 77, 679-694.

GAFFAN, D., \& HARRISON, S. (1988). Inferotemporal-frontal disconnection and fornix transection in visuomotor conditional learning by monkeys. Behavioral \& Brain Research, 31, 149-163.

Goldman, P. S., \& Rosvold, H. E. (1970). Localization of function within the dorsolateral prefrontal cortex of the rhesus monkey. Experimental Neurology, 27, 291-304.

Goldman, P. S., Rosvold, H. E., Vest, B., \& Galkin, T. W. (1971). Analysis of the delayed-alternation deficit produced by dorsolateral prefrontal lesions in the rhesus monkey. Journal of Comparative \& Physiological Psychology, 77, 212-220.

Gross, C. G., \& WEISKRANTZ, L. (1962). Evidence for dissociation of impairment on auditory discrimination and delayed response following lateral frontal lesions in monkeys. Experimental Neurology, 5, 453-476.

Hitzig, E. (1874). Untersuchungen über das Gehirn [Investigations of the brain]. Berlin: A. Hirschwald.

Hunter, W. S. (1913). The delayed reaction in animals. Behavioral Monographs, 2, 21-30.

IVERSEN, S. D., \& MishKin, M. (1970). Perseverative interference in monkeys following selective lesions of the inferior prefrontal convexity. Experimental Brain Research, 11, 376-386.

JACOBSEN, C. F. (1936). Studies of cerebral function in primates: I. The functions of the frontal association areas in monkeys. Comparative Psychology Monographs, 13, 1-60.

Kowalska, D. M., Bachevalier, J., \& Mishkin, M. (1991). The role of the inferior prefrontal convexity in performance of delayed nonmatching-to-sample. Neuropsychologia, 29, 583-600.

LEVY, R., \& GoldMAN-RAKIC, P. S. (1999). Association of storage and processing functions in the dorsolateral prefrontal cortex of the nonhuman primate. Journal of Neuroscience, 19, 5149-5158.

LEVY, R., \& GoldMAN-RAKIC, P. S. (2000). Segregation of working memory functions within the dorsolateral prefrontal cortex. Experimental Brain Research, 133, 23-32.

MiLlER, E. K. (2000). The prefrontal cortex and cognitive control. $\mathrm{Na}$ ture Reviews Neuroscience, 1, 59-65.

Miller, M. H., \& ORBACH, J. (1972). Retention of spatial alternation following frontal lobe resections in stump-tailed macaques. Neuropsychologia, 10, 291-298.

MishKIN, M. (1954). Visual discrimination performance following partial ablations of the temporal lobe: II. Ventral surface vs. hippocampus. Journal of Comparative \& Physiological Psychology, 47, 187-193.

MisHKIN, M. (1957). Effects of small frontal lesions on delayed alternation in monkeys. Journal of Neurophysiology, 20, 615-622.

Mishrin, M., \& ManNing, F. J. (1978). Non-spatial memory after selective prefrontal lesions in monkeys. Brain Research, 143, 313-323.

MishKin, M., \& Pribram, K. H. (1954). Visual discrimination performance following partial ablations of the temporal lobe: I. Ventral vs. lateral. Journal of Comparative \& Physiological Psychology, 47, 1420.

Mishin, M., \& Pribram, K. H. (1955). Analysis of the effects of frontal lesions in monkey: I. Variations of delayed alternation. Journal of Comparative \& Physiological Psychology, 48, 492-495.

MishKin, M., \& Pribram, K. H. (1956). Analysis of the effects of frontal lesions in monkey: II. Variations of delayed response. Journal of Comparative \& Physiological Psychology, 49, 36-40.
Mishrin, M., Vest, B., Waxler, M., \& Rosvold, H. E. (1969). A reexamination of the effects of frontal lesions on object alternation. Neuropsychologia, 7, 357-364.

MurRaY, E. A., BusseY, T. J., \& Wise, S. P. (2000). Role of prefrontal cortex in a network for arbitrary visuomotor mapping. Experimental Brain Research, 133, 114-129.

Ó Scalaidhe, S. P., Wilson, F. A., \& Goldman-Rakic, P. S. (1999). Face-selective neurons during passive viewing and working memory performance of rhesus monkeys: Evidence for intrinsic specialization of neuronal coding. Cerebral Cortex, 9, 459-475.

OsCar-Berman, M. (1975). The effects of dorsolateral-frontal and ventrolateral-orbitofrontal lesions on spatial discrimination learning and delayed response in two modalities. Neuropsychologia, 13, 237246.

OwEN, A. M. (2000). The role of the lateral frontal cortex in mnemonic processing: The contribution of functional neuroimaging. Experimental Brain Research, 133, 33-43.

Owen, A. M., Stern, C. E., Look, R. B., Tracey, I., Rosen, B. R., \& Petrides, M. (1998). Functional organization of spatial and nonspatial working memory processing within the human lateral frontal cortex. Proceedings of the National Academy of Sciences, 95, 7721-7726.

PARKER, A., \& GAFFAN, D. (1998). Memory after frontal/temporal disconnection in monkeys: Conditional and nonconditional tasks, unilateral and bilateral frontal lesions. Neuropsychologia, 36, 259-271.

Passingham, R. [E.] (1975). Delayed matching after selective prefrontal lesions in monkeys (Macaca mulatta). Brain Research, $\mathbf{9 2}$, 89-102.

Passingham, R. E., Toni, I., \& Rushworth, M. F. (2000). Specialization within the prefrontal cortex: The ventral prefrontal cortex and associative learning. Experimental Brain Research, 133, 103-113.

Petrides, M. (1991). Monitoring of selections of visual stimuli and the primate frontal cortex. Proceedings of the Royal Society of London: Series B, 246, 293-298.

Petrides, M. (1995). Impairments on nonspatial self-ordered and externally ordered working memory tasks after lesions of the mid-dorsal part of the lateral frontal cortex in the monkey. Journal of Neuroscience, 15, 359-375.

Petrides, M. (2000a). Dissociable roles of mid-dorsolateral prefrontal and anterior inferotemporal cortex in visual working memory. Journal of Neuroscience, 20,7496-7503.

PETRIDES, M. (2000b). The role of the mid-dorsolateral prefrontal cortex in working memory. Experimental Brain Research, 133, 44-54.

Petrides, M., \& Milner, B. (1982). Deficits on subject-ordered tasks after frontal- and temporal-lobe lesions in man. Neuropsychologia, 20, 249-262.

PeTRIDEs, M., \& PANdyA, D. N. (1994). Comparative architectonic analysis of the human and macaque frontal cortex. In F. Boller \& J. Grafman (Eds.), Handbook of neuropsychology (Vol. 9, pp. 17-58). Amsterdam: Elsevier.

Petrides, M., \& Pandya, D. N. (2002). Comparative cytoarchitectonic analysis of the human and the macaque ventrolateral prefrontal cortex and corticocortical connection patterns in the monkey. European Journal of Neuroscience, 16, 291-310.

Pribram, K. H., \& MishKIN, M. (1956). Analysis of the effects of frontal lesions in monkey: III. Object alternation. Journal of Comparative \& Physiological Psychology, 49, 41-45.

QUINTANA, J., \& FUSTER, J. M. (1992). Mnemonic and predictive functions of cortical neurons in a memory task. NeuroReport, 3, 721-724.

Quintana, J., \& Fuster, J. M. (1993). Spatial and temporal factors in the role of prefrontal and parietal cortex in visuomotor integration. Cerebral Cortex, 3, 122-132.

Quintana, J., Yajeya, J., \& Fuster, J. M. (1988). Prefrontal representation of stimulus attributes during delay tasks: I. Unit activity in cross-temporal integration of sensory and sensory-motor information. Brain Research, 474, 211-221.

RaO, S. C., RaIner, G., \& Miller, E. K. (1997, May 2). Integration of what and where in the primate prefrontal cortex. Science, 276, 821-824.

Rosenkilde, C. E., Rosvold, H. E., \& Mishin, M. (1981). Time discrimination with positional responses after selective prefrontal lesions in monkeys. Brain Research, 210, 129-144. 
Rushworth, M. F., Nixon, P. D., Eacott, M. J., \& Passingham, R. E. (1997). Ventral prefrontal cortex is not essential for working memory. Journal of Neuroscience, 17, 4829-4838.

ShINDY, W. W., Posley, K. A., \& Fuster, J. M. (1994). Reversible deficit in haptic delay tasks from cooling prefrontal cortex. Cerebral Cortex, 4, 443-450.

Stamm, J. S. (1973). Functional dissociation between the inferior and arcuate segments of dorsolateral prefrontal cortex in the monkey. Neuropsychologia, 11, 181-190.

Stamm, J. S., \& WebeR-Levine, M. L. (1971). Delayed alternation impairments following selective prefrontal cortical ablations in monkeys. Experimental Neurology, 33, 263-278.

Ungerleider, L. G., Courtney, S. M., \& Haxby, J. V. (1998). A neural system for human visual working memory. Proceedings of the National Academy of Sciences, 95, 883-890.

UNGERLEIDER, L. G., \& MishKIN, M. (1982). Two cortical visual systems. In R. J. W. Mansfield (Ed.), Analysis of visual behavior (pp. 549586). Cambridge, MA: MIT Press.

Volgushev, M., Vidyasagar, T. R., Chistiakova, M., \& Eysel, U. T. (2000). Synaptic transmission in the neocortex during reversible cooling. Neuroscience, 98, 9-22.

WALKER, A. E. (1940). A cytoarchitectural study of the prefrontal area of the macaque monkey. Journal of Comparative Neurology, 73, 5986.

Wang, M., Zhang, H., \& Li, B. M. (2000). Deficit in conditional visuomotor learning by local infusion of bicuculline into the ventral prefrontal cortex in monkeys. European Journal of Neuroscience, $\mathbf{1 2}$, 3787-3796.

Wilson, F. A., Ó Scalaidhe, S. P., \& Goldman-Rakic, P. S. (1993, June 25). Dissociation of object and spatial processing domains in primate prefrontal cortex. Science, 260, 1955-1958.

Wise, S. P., \& Murray, E. A. (2000). Arbitrary associations between antecedents and actions. Trends in Neurosciences, 23, 271-276.

\section{NOTE}

1. Other areas of the PFC are not addressed in this review for several reasons. Although in some of the earlier less precise PFC lesions area 10 was involved, this region has not been systematically studied. The term dorsolateral PRC lesions do not refer to damage to the dorsomedial portion of area 9 and area 44, which is a premotor area in the frontal cortex.

(Manuscript received August 3, 2004; revision accepted for publication November 11, 2004.) 